\title{
Leucoplasia oral: Conceptos actuales
}

\section{Oral leukoplakia: Current considerations}

\author{
Escribano-Bermejo $M^{*}$, Bascones-Martínez $A^{* *}$
}

\section{RESUMEN}

La leucoplasia es la lesión premaligna más frecuente de la cavidad oral. La Organización Mundial de la Salud la define clínicamente como una lesión predominantemente blanca de la mucosa oral que no puede caracterizarse como ninguna otra lesión conocida y con una elevada tendencia a convertirse en un cáncer oral. El objetivo de esta revisión es hacer un repaso al conocimiento actual acerca de la leucoplasia oral prestando especial atención a su nomenclatura, su etiología, su potencial maligno y su tratamiento.

Palabras clave: Leucoplasia oral, lesiones premalignas.

\section{SUMMARY}

The oral leukoplakia is the most frequent premalignancy of the oral cavity. Clinically, it was defined by the WHO as a predominantly white lesion of the oral mucosa that cannot be characterized as any other definable lesion, with an obvious tendency to become an oral cancer. The aim of this article is to review the current concepts related with the oral leukoplakia, paying special attention to terminology, aetiology, malignant potential and treatment.

Key words: Oral leukoplakia, premalignancies.

Fecha de recepción: 10 de septiembre de 2008.

Aceptado para publicación: 2 de octubre de 2008.

* $\quad$ Máster en Periodoncia e Implantes. UCM.

** Catedrático de Medicina Bucal y Periodoncia. Departamento de Estomatología III. UCM.

Escribano-Bermejo M, Bascones-Martínez A. Leucoplasia oral: Conceptos actuales. Av. Odontoestomatol 2009; 25 (2): 83-97.

\section{CONCEPTO}

El término leucoplasia fue utilizado por primera vez por E. Schwimmer a finales del siglo XIX, y procede de las palabras griegas "leuco" que significa blanco y "plakos" que significa placa.

En 1978, la Organización Mundial de la Salud (OMS) pretendió consensuar la terminología utilizada hasta el momento, y precisó su definición como una mancha blanca que no puede caracterizarse como otra entidad clínica ni patológica. La leucoplasia oral como lesión precancerosa, fue definida en Uppsala en 1994 por un grupo de expertos en patología oral, como una lesión predominantemente blanca de la mucosa oral que no puede ser caracterizada como ninguna otra lesión, ni clínica ni histopatológicamente, y que tiene tendencia a la transformación maligna (1). El hecho de que algunos carcinomas escamosos aparezcan junto a lesiones leucoplásicas y que leucoplasias orales hayan sufrido con el paso del tiempo una transformación hacia lesiones can- 
cerosas, es razón suficiente para considerar a la leucoplasia oral como una lesión premaligna.

\section{EPIDEMIOLOGÍA}

La incidencia de la leucoplasia oral ha sido evaluada longitudinalmente por estudios realizados en India y Japón. Esta incidencia está condicionada por la edad, el sexo y el hábito tabáquico de los sujetos. De esta forma, en India se encontraron incidencias anuales de 5,2/1000-30/1000 en varones fumadores, de 0,6/ $1000-5,8 / 1000$ en varones no fumadores, y de 0,2/ 1000-1,3/1000 en mujeres independientemente de su hábito tabáquico (2). En Japón, a pesar de tener hábitos muy diferentes a los existentes en India, la incidencia anual se mostró muy parecida a la observada en India: 4,1/1000 entre hombres y 0,7/1000 entre mujeres (3).

En cuanto a los datos de prevalencia de leucoplasia oral, referida a los casos identificados en una población en un momento determinado, existe un acuerdo mundial en cuanto a las cifras. Estos datos oscilan entre $1 \%-5 \%$ dependiendo del país a estudio, del tipo de población, del tipo de hábito tabáquico y de la definición clínica de leucoplasia utilizada. En una revisión sistemática de 2003, Petti y cols. agruparon datos de 17 países en los que se realizaron estudios de prevalencia entre 1986-2002. A pesar de la gran heterogeneidad de los estudios, las técnicas estadísticas utilizadas arrojaron una prevalencia mundial unificada de un 2,6\% (4).

En los países desarrollados la leucoplasia parece afectar a individuos entre la cuarta y séptima década de la vida. En países en desarrollo, la aparición de este tipo de lesiones se adelanta entre 5-10 años.

La distribución por sexos varía según la diferente distribución del hábito tabáquico, aunque puede unificarse en un 3:1 más frecuentemente en hombres que en mujeres.

La localización oral en la que se presenta la leucoplasia también tiene relación con el tipo de hábito tabáquico. Estudios realizados en India han mostrado que en sujetos fumadores, en el $85 \%$ de los casos las lesiones aparecen en mucosa vestibular o comi- suras bucales. Sin embargo, cuando se analizan los datos de los sujetos que tienen hábito de fumar invertido, la localización preferente pasa a ser el paladar, englobando un 70\% de las lesiones (5). En estudios de países desarrollados, las localizaciones más habituales son la mucosa vestibular y las comisuras orales, englobando el $90 \%$ de todas las leucoplasias orales (6).

\section{ETIOLOGÍA}

El consumo de tabaco es el factor predisponente más común en el desarrollo de leucoplasias orales, aunque una pequeña proporción de ellas no se asocian con una causa conocida (7). En los países en desarrollo, el consumo de tabaco y de la nuez de areca, juntos o combinados, son los principales responsables de la mayoría de las leucoplasias orales. Tanto es así, que el estudio longitudinal a 10 años de Gupta y cols. realizado en India concluía que de no existir el consumo de tabaco en la población a estudio, la leucoplasia sería prácticamente inexistente (2). En los países desarrollados, la importancia del tabaco en la aparición de leucoplasias orales es también muy importante, observando como los grandes fumadores tienen hasta 7 veces más riesgo de tener leucoplasia oral que los no fumadores (8). La importancia del tabaco en la etiología de la leucoplasia oral se ve confirmada por la mejoría y en muchos casos la desaparición de las lesiones con el cese del hábito tabáquico. En general, según estudios realizados en Dinamarca y Estados Unidos, entre un 50\% y un $78 \%$ de las lesiones desaparecen tras 12 meses del cese del hábito de fumar $(9,10)$.

La importancia del consumo de alcohol en la etiología de la leucoplasia oral es controvertida, si bien, en muchos casos no puede estudiarse de forma independiente al consumo de tabaco pero parece tener un efecto sinérgico con éste (7).

La evidencia del papel del virus del papiloma humano (VPH) en la etiología de la leucoplasia oral también es controvertida. En un estudio transversal reciente, se encontró VPH en el $20 \%$ de las leucoplasias orales, si bien la relación causa-efecto no puede comprobarse con este tipo de diseño de investigación (11). De igual manera, aunque aparecen en al- 
gunas lesiones de leucoplasia, no está evidenciada la relación etiológica de Candida albicans con la leucoplasia oral.

Existen algunos casos en que la etiología de la leucoplasia es desconocida. Estos casos suponen entre un $4.2 \%$ y un $26 \%$ (7) y se conocen como leucoplasias idiopáticas.

\section{VARIEDADES CLÍNICAS}

Existen dos tipos fundamentales de leucoplasias: las homogéneas y las no homogéneas, que se subdividen a su vez en eritroleucoplasias, nodulares y exofíticas. La leucoplasia homogénea es una lesión predominantemente blanca de la mucosa oral, uniformemente plana, con una fina apariencia que puede presentar grietas o hendiduras poco profundas y de consistencia no indurada. Por el contrario, una leucoplasia no homogénea es una lesión predominantemente blanca o roja (eritroleucoplasia) con una superficie irregular, nodular o exofítica (12). Las leucoplasias homogéneas suelen ser asintomáticas, el paciente a lo sumo suele referir rugosidad. El descubrimiento suele ser casual, por lo que el tiempo de evolución de la lesión en muchos casos es incierto. Las formas homogéneas pueden cursar, por el contrario, con escozor y ardor.

Especial atención merece la leucoplasia verrucosa proliferativa. Se describió por primera vez en 1985

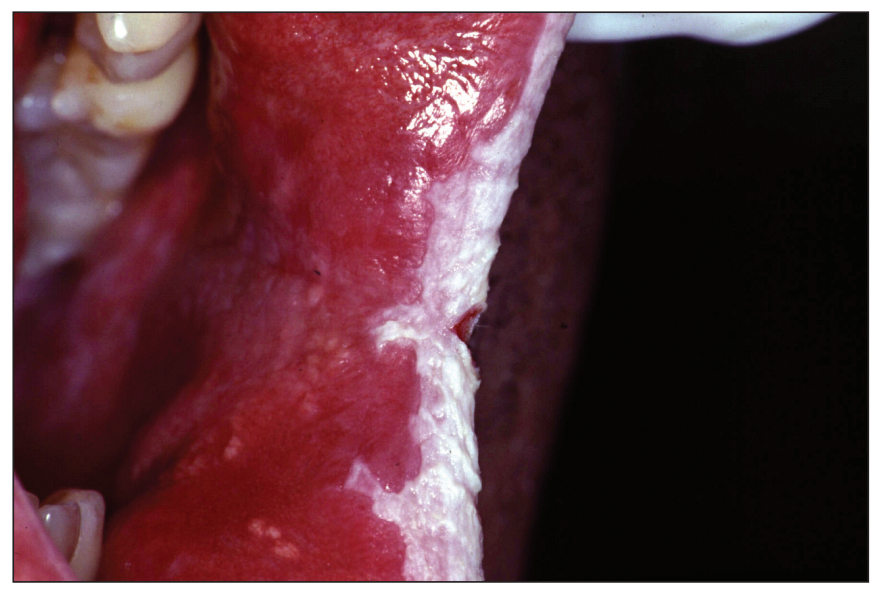

Fig. 1. Leucoplasia exofítica.

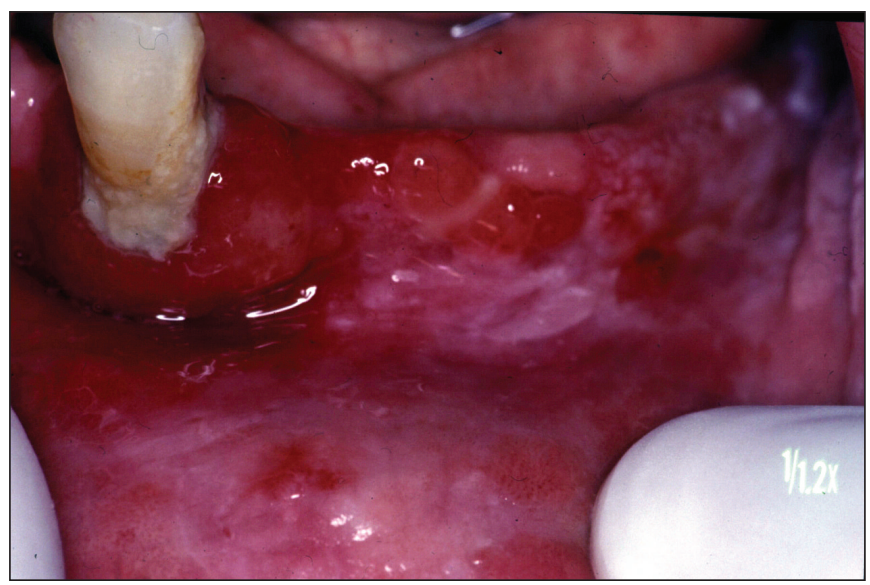

Fig 2. Eritroleucoplasia.

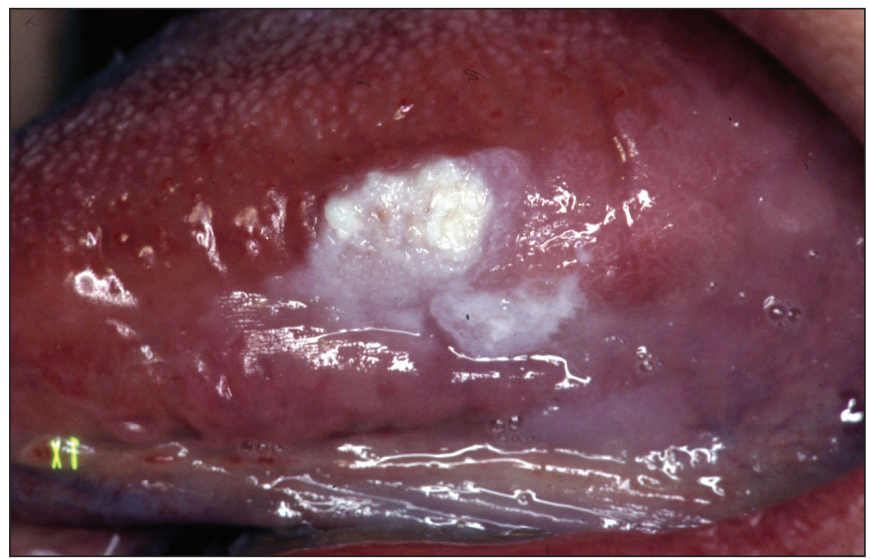

Fig 3. Leucoplasia homogénea.

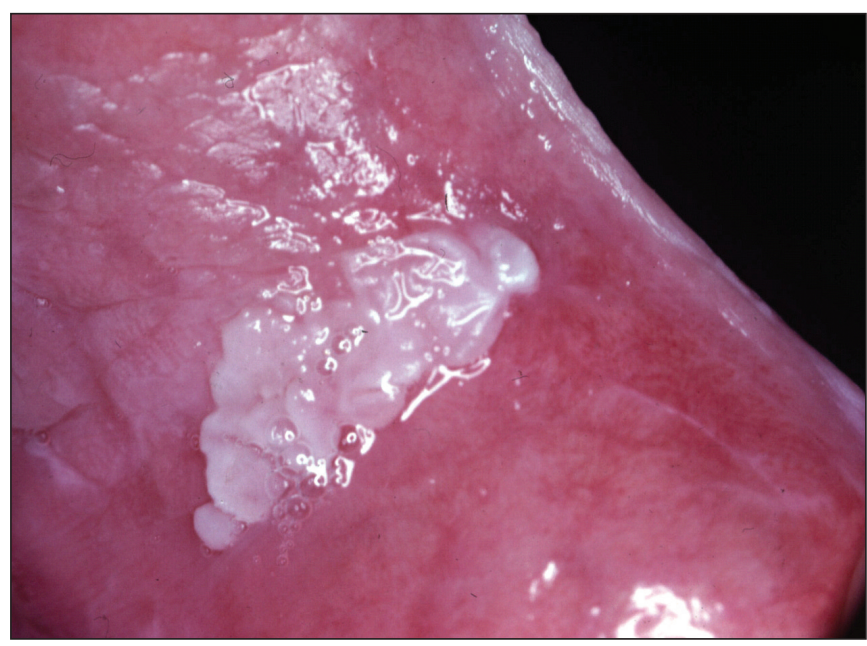

Fig. 4. Leucoplasia homogénea. 


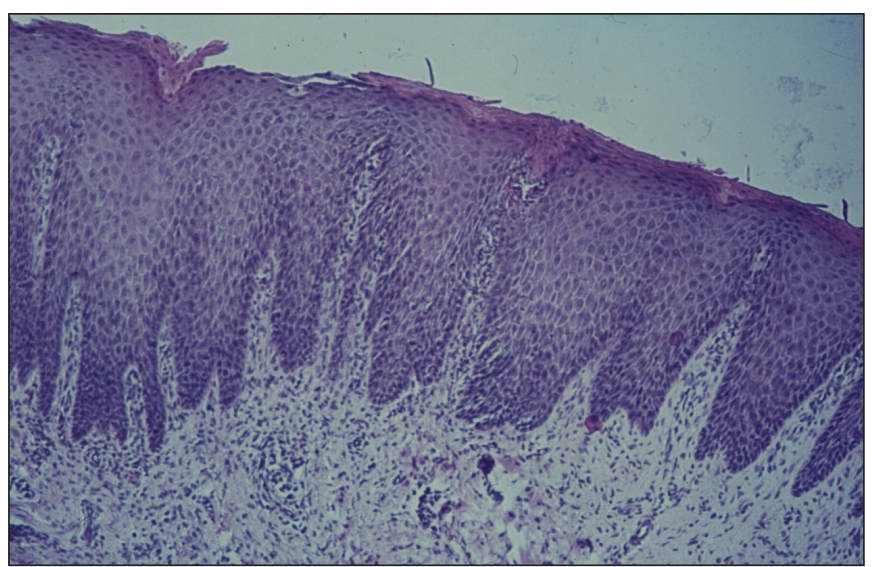

Fig. 5. Histología de una lesión de leucoplasia. Obsérvese la hiperplasia epitelial con hiperqueratosis.

(13) y se ha definido como una lesión predominantemente blanca exofítica, verrucosa, persistente, multifocal, clínicamente agresiva, resistente al tratamiento, y con gran potencial de malignización (14, 15). La proporción varón/mujer de esta lesión es de $1 / 4$, y suele darse en sujetos de edad avanzada. Se desconoce la causa de este tipo de leucoplasias, aunque esta forma clínica es la más asociada al virus del papiloma humano $(15,16)$. En muchas ocasiones es clínicamente indistinguible de un carcinoma verrucoso (17).

\section{HISTOPATOLOGÍA}

La leucoplasia puede presentar un patrón histopatológico variable, desde una hiperqueratosis sin displasia epitelial, hasta una displasia severa con fenómenos de atrofia o hiperplasia epitelial. Atendiendo a la presencia de displasia como dato fundamental, clásicamente se han distinguido dos tipos de leucoplasia: las displásicas y las no displásicas. Las leucoplasias con displasia suelen corresponderse con las formas clínicas no homogéneas y pueden estar sobreinfectadas por Candida albicans (18).

Por definición, el término de displasia hace referencia al crecimiento desordenado del epitelio. Los criterios de displasia se describen en la tabla 1. Los cambios displásicos se gradúan como displasia leve, moderada o severa. En la displasia leve los

\section{TABLA 1}

Los criterios utilizados para hacer un diagnóstico de displasia epitelial son (22):

- Pérdida de la polaridad de las células del estrato basal.

- Presencia de más de una capa de células basales con aspecto basaloide.

- Aumento de la relación núcleo/citoplasma.

- Papilas en forma de gota.

- Estratificación epitelial irregular.

- Aumento del número de mitosis, con algunas atípicas.

- Células mitóticas anormales en forma.

- Presencia de mitosis en la mitad superior del epitelio.

- Pleomorfismo celular y nuclear.

- Hipercromatismo nuclear.

- Nucléolos aumentados.

- Pérdida de la cohesión celular.

- Queratinización de células aisladas o en grupo.

cambios displásicos son «mínimos» y están confinados al tercio inferior del epitelio. En la displasia moderada los cambios displásicos ocurren en los dos tercios inferiores del epitelio. En la displasia severa los cambios ocupan más de dos tercios, pero no todo el espesor epitelial (19). El porcentaje de leucoplasias con displasia varía en gran manera en la literatura científica. Las leucoplasias con displasia pueden suponer entre en un 6-10\% (20) y $15-23 \%(21)$.

\section{DIAGNÓSTICO}

La interpretación del término leucoplasia es estrictamente clínica (sin connotaciones histológicas), y su diagnóstico se realiza respetando esta premisa, empezando por establecer una aproximación diagnóstica (diagnóstico provisional) basada en las caracte- 
rísticas morfológicas de la lesión (lesión elemental, localización, extensión, sintomatología acompañante, etc.) (23). El diagnóstico definitivo se obtendrá después de establecer unas pautas de comportamiento que conllevan la identificación y eliminación de los posibles factores etiológicos (traumáticos, micóticos, etc.), y en el control evolutivo de la lesión. En el caso de persistir ésta pasado un período de 2-4 semanas tras el cese de los posibles factores etiológicos se hará necesario el estudio histopatológico (1).

Las lesiones muy extensas requerirán la toma de varias muestras simultáneas, en las que se deberán incluir tanto áreas clínicamente sospechosas (áreas rojas, ulceradas, dolorosas o induradas) como aquéllas de apariencia benigna, debido a que en ocasiones no hay correlación entre las alteraciones histológicas y la apariencia de benignidad clínica de la lesión (23). La técnica quirúrgica y la fijación de la muestra son fundamentales para asegurar una lectura microscópica óptima. La citología y la coloración con azul de toluidina tienen un interés limitado (24). La citología fue utilizada en el pasado, pero teniendo en cuenta las dificultades que se presentan para establecer un diagnóstico con una muestra que carece de información sobre las modificaciones epiteliales profundas, la citología no debe ser utilizada en el diagnóstico de las leucoplasias (24). La coloración vital con azul de toluidina, que permite detectar zonas epiteliales displásicas, posee una baja especificidad; únicamente puede ser útil en la orientación de la biopsia o en el seguimiento de lesiones extensas que no son subsidiarias más que de un control por biopsias de repetición (24).

\section{DIAGNÓSTICO DIFERENCIAL}

El diagnóstico diferencial debe realizarse con las siguientes entidades (23):

- Candidiasis oral. El primer paso para establecer el diagnóstico diferencial de una lesión blanca en la mucosa bucal es determinar si se puede desprender (como ocurre en la candidiasis seudomembranosa) o no, mediante el empleo de una gasa, un depresor lingual u otro instrumento atraumático. En otras ocasiones el diagnóstico se realiza mediante cultivo o mediante la tinción de PAS que determinará la presencia de hifas en el epitelio.

- Liquen plano y lupus eritematoso. Si la lesión se localiza en ambas mucosas yugales, deberá establecerse el diagnóstico diferencial con el liquen plano y el lupus eritematoso. El diagnóstico definitivo se confirmará con la biopsia y el empleo de técnicas histoquímicas de inmunofluorescencia y PAS. En el caso del lupus eritematoso se requerirá además una determinación analítica complementaria.

- Lesiones traumáticas. Las lesiones traumáticas más frecuentes son la mucosa mordisqueada y la queratosis focal. Se caracterizan por el binomio causa/efecto y porque una vez eliminada la causa (por ejemplo, superficies dentarias anfractuosas) se evidenciará la mejoría de la lesión.

- Lesiones blancas hereditarias. En el nevus blanco esponjoso el antecedente familiar es un aspecto orientador muy importante y que se debe recoger en la anamnesis. Mediante el estudio histopatológico, el hallazgo de un marcado edema en el estrato espinoso confirmará el diagnóstico.

- Otras lesiones blancas que intervienen en el diagnóstico diferencial son la leucoplasia vellosa, la lengua geográfica, las quemaduras químicas, las verrugas vulgares u otras lesiones papilomatosas blancas de origen viral (23).

\section{CLASIFICACIÓN Y ESTADIAJE CLÍNICO}

Para la clasificación se distinguen dos categorías: el tamaño de la leucoplasia y los resultados histológicos, señalando la presencia o no de displasia epitelial. Con ello se realizó un intento de estadiaje de la leucoplasia oral, en cierto modo similar al del cáncer oral, pero con diferentes matices (Tabla 2).

\section{EVOLUCIÓN Y PRONÓSTICO}

La evolución de la lesión de leucoplasia oral puede estar marcada por una modificación del aspecto clínico o por una degeneración maligna. Esto hace 


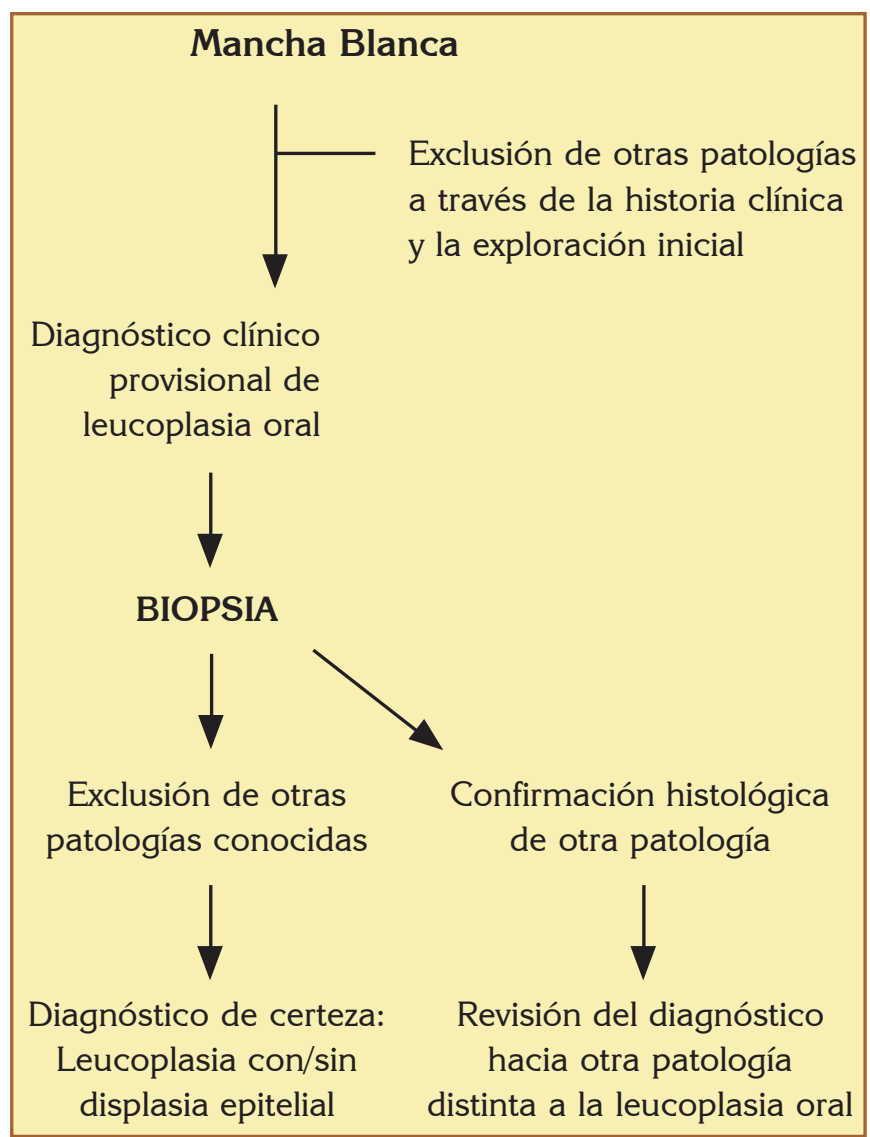

Fig. 6. Representación esquemática de los pasos a seguir en el diagnóstico de la leucoplasia oral (25).

que la vigilancia clínica e histológica sea indispensable (24).

La evolución clínica puede hacerse en el sentido de una regresión o en el de una extensión de la lesión. La leucoplasia simple regresa en un buen número de casos. La leucoplasia verrugosa puede tanto extenderse y volverse erosiva como remitir; por último, la leucoplasia erosiva aumenta de tamaño más a menudo que remite. Estas modificaciones tienen una incidencia diferente en función de la localización de la lesión y, sobre todo, en función de la supresión de los factores etiológicos (26).

\section{MALIGNIZACIÓN}

La leucoplasia oral esta considerada por la OMS como una lesión precancerosa (1), con dos circuns-

\section{TABLA 2.- CLASIFICACIÓN Y SISTEMA DE ESTADIAJE DE LA LEUCOPLASIA ORAL (1)}

L (tamaño de la leucoplasia):

- L1: leucoplasias únicas o múltiples juntas de tamaño $<2 \mathrm{~cm}$.

- L2: leucoplasias únicas o múltiples juntas de tamaño 2-4 cm.

- L3: leucoplasias únicas o múltiples juntas de tamaño $\geq 4 \mathrm{~cm}$.

- Lx: tamaño no especificado.

P (patólogo):

- P0: sin displasia epitelial (incluye "quizá displasia epitelial leve").

- P1: con displasia epitelial (incluye displasia epitelial "leve a moderada").

- Px: ausencia o presencia de displasia epitelial no especificada en el informe del patólogo.

S (sistema de estadiaje):

- OLEP.

- Estadio I: L1P0.

- Estadio II: L2PO.

- Estadio III: L3P0 o L1L2P1.

- Estadio IV: L3P1.

Reglas generales para el sistema de estadiaje de la leucoplasia oral:

- Si existen dudas sobre en qué categoría L o P incluir un determinado caso, se seleccionará la categoría menos avanzada.

- Lo mismo sucederá respecto al sistema de estadiaje.

- En los casos en que existan múltiples biopsias de leucoplasias únicas múltiples se empleará el resultado del patólogo más severo.

- Se debería anotar la localización de acuerdo con el ICD-DA para futuras publicaciones de los resultados.

tancias que ratifican esta apreciación. La primera es que en las proximidades de gran número de carcinomas epidermoides se han diagnosticado vestigios de leucoplasias (27). En segundo lugar, se ha podido 
observar que con el paso del tiempo algunas leucoplasias han malignizado (22). Además, ambas entidades patológicas comparten cambios morfológicos y citológicos observados en carcinomas in situ, y comparten alteraciones cromosómicas, genómicas y moleculares detectadas en cánceres orales invasivos (25).

El porcentaje estimado de transformación maligna varía en función del país en donde se haya realizado el estudio y de dónde y cómo se haya seleccionado la muestra, bien de un centro hospitalario, bien de una muestra aleatoria. El rango de transformación maligna de la leucoplasia oral oscila según los estudios $(9,28)$, entre el $0,13 \%$ y el $17,5 \%$.

Diferentes estudios en los que se ha realizado un seguimiento de pacientes con leucoplasia, han puesto de manifiesto que existen unos factores clínicos, histopatológicos y moleculares relacionados con un mayor riesgo potencial de transformación maligna de una leucoplasia.

\section{FACTORES CLÍNICOS}

\section{Apariencia clínica}

Según los estudios, las leucoplasias no homogéneas tienen un potencial de malignización de entre 4 a 5 veces mayor que el de las leucoplasias homogéneas.
Esto no resta importancia al control y seguimiento de las leucoplasias homogéneas, que han mostrado tasas de malignización de un 0,6-5\% $(9,37)$.

\section{Etiología}

\section{Tabaco}

Las leucoplasias asociadas con el consumo de tabaco parecen tener menor potencial maligno que el de las no asociadas a este hábito. Según Silverman y cols., esta diferencia oscila en una tasa de malignización de $12 \%$ en fumadores y de $32 \%$ en no fumadores (9). Estos resultados son llamativos, ya que el tabaco es el factor etiológico más importante del carcinoma de la mucosa oral. Parece, además, que en fumadores, la leucoplasia suele encontrarse en suelo de boca, mientras que en no fumadores la localización más frecuente suelen ser los bordes laterales de la lengua (38).

\section{Infección por Candida}

La asociación entre la infección por Candida y el riesgo de transformación maligna de la leucoplasia se originó cuando se relacionó Candida con la leucoplasia no homogénea y con la presencia de displasia epitelial. En estudios en animales, el género Candida ha sido capaz de producir carcinomas en la

\section{TABLA 3.- ESTUDIOS SELECCIONADOS SOBRE TRANSFORMACIÓN DE LA LEUCOPLASIA} ORAL (29)

\begin{tabular}{|l|c|c|c|c|}
\hline Autor y año & País & Muestra & $\begin{array}{c}\text { Seguimiento } \\
\text { (años) }\end{array}$ & $\begin{array}{c}\text { \% de transformación } \\
\text { maligna }\end{array}$ \\
\hline Pindborg et al, 1968 (30) & Dinamarca & 248 & 3,9 & 4,4 \\
Silverman E Rosen, 1968 (31) & EEUU & 117 & $1-11$ & 6,0 \\
Kramer et al, 1970 (32) & Reino Unido & 187 & $?$ & 4,8 \\
Mehta et al, 1972 (33) & India & 117 & 10 & 0,9 \\
Silverman et al, 1976 (28) & India & 4.762 & 2 & 0,13 \\
Bánóczy et al, 1984 (34) & Hungría & 670 & 9,8 & 6,0 \\
Silverman et al, 1984 (9) & EEUU & 257 & 7,2 & 17,5 \\
Lind, 1987 (35) & Noruega & 157 & 9,3 & 8,9 \\
Schepman et al, 1998 (36) & Holanda & 166 & 2,5 & 12,0 \\
\hline
\end{tabular}


mucosa oral de ratas a través de la producción de nitrosaminas carcinogénicas.

\section{Otros factores}

El virus del papiloma humano (VPH) ha sido muy estudiado en relación a la transformación maligna de la leucoplasia oral. Los resultados de los estudios varían ampliamente y son inconsistentes. En un metaanálisis publicado en 2001 se concluyó que la aparición del virus del papiloma humano era de 2-3 veces más frecuente en lesiones precancerosas, y de 4-5 veces más frecuente en carcinoma oral de células escamosas que en epitelio normal (39). Sin embargo, estos estudios no son suficientes para establecer un papel etiológico del VPH en la transformación maligna de la leucoplasia oral (29).

\section{Localización}

Algunos autores han señalado que las lesiones de leucoplasia que acontecen en suelo de boca o en la cara ventral de la lengua tienen un mayor potencial maligno que las leucoplasias de otras regiones de la mucosa oral (40). Sin embargo, este hecho no ha sido corroborado por otros estudios (36). Es posible que el concepto de localizaciones de alto riesgo para la leucoplasia oral gane apoyo a través de los estudios genéticos (41).

\section{FACTORES HISTOLÓgICOS}

La displasia epitelial, definida como una lesión del epitelio escamoso estratificado caracterizada por una atipia celular y una maduración y estratificación anormales del epitelio, ha mostrado poseer tasas de malignización diferentes en función del grado en el que se dé ésta en el epitelio. Así, la displasia inicial, moderada y severa, evoluciona, según algunos autores, hacia carcinomas en el 3\%, 4\%, y 43\% de los casos (tomado de Burkhardt E Maeker 1978 en (29)). Sin embargo, la literatura no es concluyente a este respecto (29). Además, existe una gran variabilidad interexaminador a la hora de definir los grados de displasia. Es por ello que en el último consenso mundial de medicina oral y en la última revisión publicada en 2008, se concluye que se necesitan estudios longitudinales que evalúen la fiabilidad en la valoración de la displasia como factor pronóstico en la malignización de las lesiones precancerosas $(42,43)$. Los problemas que se presentan en la evaluación del significado de la displasia epitelial se deben a la falta de criterios objetivos establecidos, a la división más o menos arbitraria de sus grados de severidad, a la falta de criterios de calibración entre examinadores y a la falta de conocimiento sobre los criterios de displasia que son más importantes a la hora de predecir la transformación maligna de una lesión (44).

\section{Factores moleculares}

En los últimos años se han publicado numerosos estudios acerca de la aplicación de marcadores de biología molecular para la determinación del riesgo de cáncer (29). Existen dos formas básicas por las que se ha estudiado este hecho en el pasado. En algunos estudios transversales se ha intentado comparar la presencia y distribución de estos marcadores en lesiones precancerosas y en lesiones de carcinoma in situ. La otra forma en que esto ha sido estudiado en el pasado ha sido a través de estudios retrospectivos, en los que se han comparado los marcadores moleculares de lesiones que posteriormente dieron lugar a lesiones malignas con los de lesiones que permanecieron en el tiempo sin malignizar. Es evidente, por tanto, que aún se necesitan estudios prospectivos que evalúen estos marcadores de riesgo de malignización (29).

Los marcadores moleculares mejor estudiados en la actualidad son:

1. Marcadores genómicos; entre los que se incluyen alteraciones cromosómicas, cambios en la expresión de los oncogenes y en genes supresores de tumores.

2. Marcadores de proliferación celular.

3. Marcadores de diferenciación celular, entre los que se incluyen las queratinas y los antígenos de hidratos de carbono.

La aneuploidía del DNA supone la pérdida de la diploidía de éste y, por tanto, la inestabilidad genética 
y la aparición de un DNA aberrante. En cánceres de otras regiones del organismo se ha mostrado como un factor pronóstico importante de su evolución. Sin embargo, en el estudio de la leucoplasia oral existe controversia en cuanto a su asociación con la displasia epitelial. Existen una serie de estudios de los últimos años que apuntan que la aneuploidía del DNA es un potente predictor de la malignización de la leucoplasia oral y de la eritroplasia $(45,46)$.

La pérdida de la heterocigosidad en regiones que contienen genes supresores de tumores también ha sido estudiada en relación a la malignización de lesiones precancerosas. La pérdida de material genético en uno de los cromosomas que forman el par tiene mayor o menor relevancia en función de en qué posición se produzca dicha pérdida. Por ejemplo, la pérdida de heterocigosidad en los brazos 3p y 9p se ha mostrado asociada a la evolución hacia la malignización de lesiones precancerosas (con un riesgo de 3.8 veces más de sufrir una degeneración maligna). Además, si a estas posiciones se añade la pérdida de material genético en otras (por ejemplo: $4 q, 8 p, 11 q, 13 q, 17 q)$ el riesgo se multiplica (hasta en 33 veces) (29).

La mutación del gen supresor de tumores p53 es el cambio genético más frecuente que acontece en el desarrollo de un cáncer. Este gen se encuentra inactivado en lesiones de carcinoma oral de células escamosas, y está evidenciado que su forma mutada tiene una vida media mayor que la de la forma salvaje (29). Según Lippman y cols. la expresión de p53 se da en el $90 \%$ de las leucoplasias orales, y parece estar ausente en la mucosa normal (47). Según datos de un metaanálisis reciente, el $47 \%$ de las lesiones premalignas orales expresarían la proteína de p53. Aunque la literatura no es concluyente a la hora de definir el papel de p53 en la malignización de la leucoplasia, algunos estudios defienden que no es la simple expresión de p53 sino su patrón de distribución lo que puede determinar diferencias. Cruz y cols. encontraron que la expresión parabasal clara de 553 podría ser un indicador de desarrollo de carcinoma, incluso ante la ausencia de displasia clara en el epitelio (48). Además de p53 se han estudiado recientemente otros genes de proliferación celular y apoptosis como Ki67, D1 cyclina, p27 y p63, aunque los resultados no tienen aún capaci- dad predictiva para el pronóstico de las leucoplasias orales (49).

En cuanto a los marcadores de diferenciación celular, aún no existe literatura suficiente que defina el papel de éstos en la malignización de la leucoplasia oral.

Para concluir este apartado podemos decir que los marcadores clínicos, histológicos y moleculares pueden contribuir a evaluar el riesgo de un paciente para padecer un cáncer; sin embargo, actualmente no se dispone de un solo factor predictivo, clínicamente útil y basado en la evidencia, de transformación maligna para las leucoplasias displásicas y no displásicas (50).

\section{TRATAMIENTO}

El tratamiento de la leucoplasia tiene tres niveles de actuación, que deben adecuarse en función de los hábitos del paciente, de las sobreinfecciones asociadas, del tipo clínico, de la localización y de las características histopatológicas de la lesión (24).

\section{Modificación de hábitos del paciente}

El primer paso consiste en actuar sobre los factores desencadenantes o asociados a la leucoplasia. Deben eliminarse los elementos que puedan ser el origen de microtraumatismos mecánicos relacionados con la lesión (superficies dentarias rugosas, prótesis mal ajustadas, etc.). Si el paciente es fumador y/o consumidor habitual de bebidas alcohólicas se le recomendará que abandone estos hábitos. Aunque la remisión suele ser lenta y en ocasiones no completa, muchas leucoplasias mejoran sólo con estas medidas. Según la literatura, entre un 50-60\% de las leucoplasias desencadenadas por fumar desaparecen a los 6-12 meses de abandonar el hábito tabáquico (10).

\section{Tratamiento médico}

Si existe sospecha de una infección por Candida se establecerá un tratamiento antimicótico tópico con nistatina o miconazol durante 15 días, hasta el día programado para la revisión del paciente y la práctica de la biopsia. Si se detecta la persistencia de 
Candida en el tejido biopsiado, se pautará fluconazol u otro derivado azólico por vía oral durante 15 días más. Tras el tratamiento antimicótico, algunas leucoplasias cambian de forma clínica, evolucionando favorablemente hacia una hiperqueratosis o disminuyendo su grado de displasia.

\section{Tratamiento tópico}

Los principios activos más utilizados en aplicación tópica son el ácido retinoico (13 cis-retinoico o etretionato) y la bleomicina. El ácido retinoico debe aplicarse en orabase al 0,1\% de 3-4 veces al día (51).

El sulfato de bleomicina se pauta al $1 \%$ en dimetilsulfóxido. Su aplicación se realiza con torundas de algodón (manteniéndose 5 minutos en contacto), una vez al día, durante 2 semanas $(52,53)$. Con este tratamiento se consigue una reducción de la lesión y una disminución o desaparición de la displasia entre el 50-95\% de los pacientes tratados $(54,55)$. Estudios comparativos entre la eficacia de la exéresis quirúrgica y la utilización de bleomicina tópica han puesto de manifiesto que el índice de recurrencias es menor con la bleomicina (55).

\section{Tratamiento sistémico}

Para el tratamiento sistémico de la leucoplasia se han utilizado por vía oral la vitamina A, o sus derivados, y la vitamina E.

La vitamina A tiene dos grandes inconvenientes que limitan su administración. El primero es la necesidad de utilizar dosis elevadas para obtener alguna eficacia (100.000-300.000 U/día), con las que se manifiestan efectos secundarios importantes (exantema, sequedad de piel, prurito, etc.), además de tener un alto potencial carcinogénico. El segundo inconveniente es el elevado porcentaje de recidivas tras finalizar el tratamiento (38-55\% de los casos). En ocasiones se ha administrado en asociación con la vitamina E (100.000 U/día).

El ácido 13 cis-retinoico (isotreotinoína) se ha mostrado eficaz a dosis bajas y con una mínima toxicidad. La dosis inicial de tratamiento es de 0,2 mg/kg/ día durante 3 meses. Posteriormente se incrementa con $0,2 \mathrm{mg} / \mathrm{kg} /$ día adicionales en 3 ciclos sucesivos. No se debe superar la cantidad de $0,8 \mathrm{mg} / \mathrm{kg} /$ día si se administra durante largos períodos de tiempo, ya que podrían aparecer efectos secundarios como alteraciones cutáneas, mucosas y hematológicas (aumento de la concentración de triglicéridos y colesterol), todos ellos reversibles (58). Debido a su teratogenicidad, se recomienda utilizar una anticoncepción eficaz durante el tratamiento y al menos un mes después de suspender el tratamiento con isotretinoína.

El etretinato se emplea pautado de 1-1,5 mg/kg/día en 3 tomas, durante 3-4 meses. Es hepatotóxico y provoca prurito, sequedad de mucosas y alopecia. A dosis de $30 \mathrm{mg}$ /día durante 3-6 meses se han obtenido buenos resultados, observándose mejorías en el $70 \%$ de los casos (71).

La fenretinida (4-HPR), otro retinoide sintético, se ha utilizado como quimioprevención asociada al tratamiento quirúrgico. A dosis de 200 mg/4 veces al día es eficaz en la prevención de recidivas y de nuevas lesiones (59).

La vitamina $\mathrm{E}$ se ha empleado a dosis de 800 U/día en períodos extensos (de 6-24 meses), si bien, sólo se han apreciaron mejorías en menos de la mitad de los pacientes del estudio (60).

\section{Tratamiento quirúrgico}

En las leucoplasias con displasia, la técnica más recomendada es la cirugía con control de los márgenes (51). Su porcentaje de recurrencia se estima entre $10-20 \%$ y está en relación con la localización en suelo de boca y con la presencia de displasia (61).

El láser de $\mathrm{CO}_{2}$ se utiliza a diferentes potencias: en las leucoplasias homogéneas oscilan entre 5-8 W de energía de forma focalizada o desfocalizada (62); en las verrucosas la vaporización se hace focalizada a 10-15 W, y en las formas erosivas se utilizan 20-25 W focalizados (23). Algunos autores defienden la utilización de esta técnica por su inocuidad, la facilidad de aplicación, y por su escasa morbilidad y recurrencia, que según algunos estudios fue inferior al $10 \%$. En este mismo estudio, sólo $1,1 \%$ de las lesiones 
sufrieron una transformación maligna durante el período de seguimiento (1-219 meses) (63).

En cuanto a la crioterapia, su ventaja radica en la escasa profundidad de su campo de acción, lo que supone la aparición de cicatrices más superficiales y flexibles (80). Se han publicado tasas de recurrencia menores al 25\% $(64,65)$.

Una revisión sistemática realizada recientemente concluye que no se demostró que ninguno de los tratamientos estudiados fuera efectivo para la prevención de la transformación maligna de las leucoplasias. Algunos tratamientos fueron efectivos para la curación de las lesiones; sin embargo, no parecieron capaces de prevenir las recidivas y la malignización de la lesión. Por este motivo, los autores argumentan que la curación clínica de la leucoplasia después de su tratamiento no libera al clínico de la responsabilidad de hacer un seguimiento regular (50).

Además, según las conclusiones de esta misma revisión, aunque la cirugía sigue siendo la primera opción para la mayoría de los clínicos, no puede evaluarse la efectividad real de dicho tratamiento porque no fue posible encontrar algún ensayo controlado aleatorio que comparara la cirugía frente a ningún tratamiento (espera y observación) en la prevención de la transformación maligna de la leucoplasia; el único ensayo controlado aleatorio comparaba dos técnicas láser. Se necesitan estudios para comprobar la eficacia de la cirugía en el tratamiento de la leucoplasia. Hasta que las pruebas definitivas sobre el valor predictivo de los marcadores biológicos específicos estén disponibles, la transformación maligna debe considerarse el mejor resultado a tener en cuenta al evaluar la efectividad de los tratamientos para la leucoplasia. Se necesitan más investigaciones para evaluar el efecto que tiene sobre la malignización de la leucoplasia el hecho de eliminar el factor de riesgo (50).

\section{Evaluación y seguimiento}

El tratamiento de la leucoplasia incluye un seguimiento de las lesiones y una evaluación de las posibles recidivas que acontezcan. Si la forma de leuco- plasia no es displásica, la reevaluación clínica debe hacerse cada 6 meses. En las leucoplasia con displasia la revisión se llevará a cabo cada 2-3 meses (66). Si se producen cambios clínicos que incrementen la gravedad de la lesión o si persisten hábitos nocivos en el paciente, durante este período de seguimiento deben practicarse controles histopatológicos periódicos (23) (Tabla 4).

\section{TABLA 4.- FORMA DE REGISTRAR EL RESULTADO DEL TRATAMIENTO DE LAS LEUCOPLASIAS ORALES [Modificado por Warnakulasuriya y cols. (25) del original de Miller y cols. (67)]}

1. Tipo de tratamiento:

- Quirúrgico (incluido el láser de $\mathrm{CO}_{2}$ ).

- Químicos.

- Sólo observación y seguimiento.

2. Nivel de respuesta (en caso de tratamiento no quirúrgico o de seguimiento sin tratamiento):

- No respuesta (lesión estable).

- Respuesta parcial (reducción en tamaño $>50 \%$, pero no completa).

- Respuesta completa.

- Lesión progresiva (crecimiento en tamaño $>25 \%$ o aparición de nueva lesión).

3. Recurrencia (aparición de una nueva leucoplasia en la misma localización, independientemente del intervalo de tiempo transcurrido desde la primera lesión).

4. Nueva lesión primaria (aparición de una nueva leucoplasia en distinta localización).

5. Transformación maligna.

6. Lesión maligna en la región de cabeza y cuello.

7. Lesión maligna fuera de la región de cabeza y cuello.

8. Duración del seguimiento.

\section{BIBLIOGRAFÍA}

1. Axell T, Pindborg JJ, Smith CJ, van der Waal I. Oral white lesions with special reference to 
precancerous and tobacco- related lesions: conclusions of an international symposium held in Uppsala, Sweden, May 18-21 1994. International Collaborative Group on Oral White Lesions. J Oral Pathol Med. 1996 Feb;25(2): 4954.

2. Gupta PC, Mehta FS, Daftary DK, Pindborg JJ, Bhonsle RB, Jalnawalla PN, et al. Incidence rates of oral cancer and natural history of oral precancerous lesions in a 10-year follow-up study of Indian villagers. Community dentistry and oral epidemiology. 1980;8(6):283-333.

3. Nagao T, Ikeda N, Fukano H, Hashimoto S, Shimozato K, Warnakulasuriya S. Incidence rates for oral leukoplakia and lichen planus in a Japanese population. J Oral Pathol Med. 2005 Oct;34(9):532-9.

4. Petti S. Pooled estimate of world leukoplakia prevalence: a systematic review. Oral oncology. 2003 Dec;39(8):770-80.

5. Mehta FS, Pindborg JJ, Gupta PC, Daftary DK. Epidemiologic and histologic study of oral cancer and leukoplakia among 50,915 villagers in India. Cancer. 1969 Oct;24(4):832-49.

6. Axell T. Occurrence of leukoplakia and some other oral white lesions among 20,333 adult Swedish people. Community dentistry and oral epidemiology. 1987 Feb;15(1):46-51.

7. Napier SS, Speight PM. Natural history of potentially malignant oral lesions and conditions: an overview of the literature. J Oral Pathol Med. 2008 Jan;37(1):1-10.

8. Baric JM, Alman JE, Feldman RS, Chauncey HH. Influence of cigarette, pipe, and cigar smoking, removable partial dentures, and age on oral leukoplakia. Oral surgery, oral medicine, and oral pathology. 1982 Oct;54(4):424-9.

9. Silverman S, Jr., Gorsky M, Lozada F. Oral leukoplakia and malignant transformation. A follow-up study of 257 patients. Cancer. 1984 Feb 1;53(3):563-8.
10. Roed-Petersen B. Effect on oral leukoplakia of reducing or ceasing tobacco smoking. Acta dermato-venereologica. 1982;62(2):164-7.

11. Campisi G, Giovannelli L, Arico P, Lama A, Di Liberto C, Ammatuna P, et al. HPV DNA in clinically different variants of oral leukoplakia and lichen planus. Oral surgery, oral medicine, oral pathology, oral radiology, and endodontics. 2004 Dec;98(6):705-11.

12. Bagan JV SC. Cáncer oral: epidemiología y prevención. In: Cuenca E BP, ed. Odontología Preventiva y Comunitaria. Barcelona: Masson. 2005:195-210.

13. Hansen LS, Olson JA, Silverman S, Jr. Proliferative verrucous leukoplakia. A long-term study of thirty patients. Oral surgery, oral medicine, and oral pathology. 1985 Sep;60(3):285-98.

14. Zakrzewska JM, Lopes V, Speight P, Hopper C. Proliferative verrucous leukoplakia: a report of ten cases. Oral surgery, oral medicine, oral pathology, oral radiology, and endodontics. 1996 Oct;82 (4):396-401.

15. Aguado A BM, Seoane JM, Vázquez J, Asenjo JM. Leucoplasia verrucosa proliferativa. 1995; Rev Esp Cirug Oral Maxilofac. Rev Esp Cirug Oral Maxilofac; 17:109-12.

16. Palefsky JM, Silverman S, Jr., Abdel-Salaam M, Daniels TE, Greenspan JS. Association between proliferative verrucous leukoplakia and infection with human papillomavirus type 16. J Oral Pathol Med. 1995 May;24(5):193-7.

17. Camacho Alonso. F. LJP. Abordaje clinicoterapéutico de la leucoplasia oral. Rev Clin Esp 2004; 204(8):420-1.

18. Bascones Martínez A CLR, Esparza Gómez GC. Lesiones precancerosas de la mucosa bucal: leucoplasia, liquen plano y úlcera traumática. In: A B, ed. Tratado de odontología. Madrid: Smithkline Beechmam 1998:3073-89.

19. Lumerman H, Freedman P, Kerpel S. Oral epithelial dysplasia and the development of 
invasive squamous cell carcinoma. Oral surgery, oral medicine, oral pathology, oral radiology, and endodontics. 1995 Mar;79(3):321-9.

20. Bagán JV PA, Vera-Sempere F. Leucoplasia bucal. Estudio clínico de 33 pacientes. Rev Esp Estomatol. 1985;33:195-204.

21. Banoczy J, Csiba A. Occurrence of epithelial dysplasia in oral leukoplakia. Analysis and followup study of 12 cases. Oral surgery, oral medicine, and oral pathology. 1976 Dec;42(6):766-74.

22. Pindborg JJ. Cáncer y precáncer bucal. $1^{\mathrm{a}}$ ed. Buenos Aires: Panamericana. Cáncer y precáncer bucal. Buenos Aires Panamericana 1981.

23. García-Pola Vallejo. MJ GMJ. Oral leukoplasia. Aten Primaria 2002;29:39-49.

24. Iriarte-Ortabea. JI. CBC, Reychlerb. H. Leucoplasia bucal. Med Clin (Barc). 1996;106(10):387-94.

25. Warnakulasuriya S, Johnson NW, van der Waal I. Nomenclature and classification of potentially malignant disorders of the oral mucosa. J Oral Pathol Med. 2007 Nov;36(10):575-80.

26. Mehta FS, Pindborg JJ. Spontaneous regression of oral leukoplakias among Indian villagers in a 5 -year follow-up study. Community dentistry and oral epidemiology. 1974;2(2):80-4.

27. Bouquot JE, Weiland LH, Kurland LT. Leukoplakia and carcinoma in situ synchronously associated with invasive oral/oropharyngeal carcinoma in Rochester, Minn., 1935-1984. Oral surgery, oral medicine, and oral pathology. 1988 Feb;65(2): 199-207.

28. Silverman S, Bhargava K, Smith LW, Malaowalla AM. Malignant transformation and natural history of oral leukoplakia in 57,518 industrial workers of Gujarat, India. Cancer. 1976 Oct;38(4):1790-5.

29. Reibel J. Prognosis of oral pre-malignant lesions: significance of clinical, histopathological, and molecular biological characteristics. Crit Rev Oral Biol Med. 2003;14(1):47-62.
30. Pindborg JJ, Jolst O, Renstrup G, Roed-Petersen B. Studies in oral leukoplakia: a preliminary report on the period pervalence of malignant transformation in leukoplakia based on a followup study of 248 patients. Journal of the American Dental Association (1939). 1968 Apr;76(4): 767-71.

31. Silverman S, Jr. Observations on the clinical characteristics and natural history of oral leukoplakia. Journal of the American Dental Association (1939). 1968 Apr;76(4):772-7.

32. Kramer IR, Lucas RB, el-Labban N, Lister L. The use of discriminant analysis for examining the histological feathers of oral keratoses and lichen planus. British journal of cancer. 1970 Dec;24(4): 673-83.

33. Mehta FS, Shroff BC, Gupta PC, Daftary DK. Oral leukoplakia in relation to tobacco habits. A tenyear follow-up study of Bombay policemen. Oral surgery, oral medicine, and oral pathology. 1972 Sep;34(3):426-33

34. Banoczy J, Boros S. (25-year history of the Clinic of Preventive Dentistry at the Semmelweis University of Medicine). Fogorvosi szemle. 1984 Nov;77(11):321-3.

35. Lind PO. Malignant transformation in oral leukoplakia. Scandinavian journal of dental research. 1987 Dec;95(6):449-55.

36. Schepman KP, van der Meij EH, Smeele LE, van der Waal I. Malignant transformation of oral leukoplakia: a follow-up study of a hospital-based population of 166 patients with oral leukoplakia from The Netherlands. Oral oncology. $1998 \mathrm{Jul}$; 34(4):270-5.

37. Gupta PC, Bhonsle RB, Murti PR, Daftary DK, Mehta FS, Pindborg JJ. An epidemiologic assessment of cancer risk in oral precancerous lesions in India with special reference to nodular leukoplakia. Cancer. 1989 Jun 1;63(11):2247-52.

38. Schepman KP, Bezemer PD, van der Meij EH, Smeele LE, van der Waal I. Tobacco usage in 
relation to the anatomical site of oral leukoplakia. Oral diseases. 2001 Jan;7(1):25-7.

39. Miller CS, Johnstone BM. Human papillomavirus as a risk factor for oral squamous cell carcinoma: a meta-analysis, 1982-1997. Oral surgery, oral medicine, oral pathology, oral radiology, and endodontics. 2001 Jun;91(6):622-35.

40. Kramer IR, El-Labban N, Lee KW. The clinical features and risk of malignant transformation in sublingual keratosis. British dental journal. 1978 Mar 21;144(6):171-80.

41. Zhang L, Cheung KJ, Jr., Lam WL, Cheng X, Poh C, Priddy R, et al. Increased genetic damage in oral leukoplakia from high risk sites: potential impact on staging and clinical management. Cancer. 2001 Jun 1;91(11):2148-55.

42. Warnakulasuriya S, Reibel J, Bouquot J, Dabelsteen E. Oral epithelial dysplasia classification systems: predictive value, utility, weaknesses and scope for improvement. J Oral Pathol Med. 2008 Mar;37(3):127-33.

43. Brennan M, Migliorati CA, Lockhart PB, Wray D, Al-Hashimi I, Axell T, et al. Management of oral epithelial dysplasia: a review. Oral surgery, oral medicine, oral pathology, oral radiology, and endodontics. 2007 Mar;103 Suppl:S19 e1-2.

44. Warnakulasuriya S. Histological grading of oral epithelial dysplasia: revisited. The Journal of pathology. 2001 Jul;194(3):294-7.

45. Sudbo J, Kildal W, Risberg B, Koppang HS, Danielsen HE, Reith A. DNA content as a prognostic marker in patients with oral leukoplakia. The New England journal of medicine. 2001 Apr 26;344(17):1270-8.

46. Sudbo J, Reith A. When is an oral leukoplakia premalignant? Oral oncology. 2002 Dec;38(8): 813-4; author reply 1-2.

47. Lippman SM, Shin DM, Lee JJ, Batsakis JG, Lotan R, Tainsky MA, et al. p53 and retinoid chemoprevention of oral carcinogenesis. Cancer research. 1995 Jan 1;55(1):16-9.

48. Cruz IB, Snijders PJ, Meijer CJ, Braakhuis BJ, Snow GB, Walboomers JM, et al. p53 expression above the basal cell layer in oral mucosa is an early event of malignant transformation and has predictive value for developing oral squamous cell carcinoma. The Journal of pathology. 1998 Apr;184(4):360-8.

49. Kovesi G, Szende B. Prognostic value of cyclin D1, p27, and p63 in oral leukoplakia. J Oral Pathol Med. 2006 May;35(5):274-7.

50. Lodi G, Sardella A, Bez C, Demarosi F, Carrassi A. Interventions for treating oral leukoplakia. Cochrane database of systematic reviews (Online). 2006(4):CD001829.

51. Bagán J. Lesiones y estados precancerosos. Medicina Oral. Barcelona: Masson 1995:166-76.

52. Wong F, Epstein J, Millner A. Treatment of oral leukoplakia with topical bleomycin. A pilot study. Cancer. 1989 Jul 15;64(2):361-5.

53. Hammersley N, Ferguson MM, Rennie JS. Topical bleomycin in the treatment of oral leukoplakia: a pilot study. The British journal of oral $\mathcal{E}$ maxillofacial surgery. 1985 Aug;23(4): 251-8.

54. Epstein JB, Gorsky M, Wong FL, Millner A. Topical bleomycin for the treatment of dysplastic oral leukoplakia. Cancer. 1998 Aug 15;83(4):629-34.

55. Malmstrom M HJ, Sane J, Sysmalainen M Topical treatment of oral leukoplakia with bleomicyn. The British journal of oral $\mathcal{E}$ maxillofacial surgery. 1998;26:491-8.

56. Toma S, Benso S, Albanese E, Palumbo R, Cantoni E, Nicolo G, et al. Treatment of oral leukoplakia with beta-carotene. Oncology. 1992; 49(2):77-81.

57. Sherman JA, Partridge M. Expression of retinoic acid receptors in normal, dysplastic and 
malignant oral epithelia. The British journal of oral $\mathcal{E}$ maxillofacial surgery. 1997 Aug;35(4): 260-6.

58. Toma S, Mangiante PE, Margarino G, Nicolo G, Palumbo R. Progressive 13-cis-retinoic acid dosage in the treatment of oral leukoplakia. European journal of cancer. 1992 Oct;28B(2): 121-3.

59. Chiesa F, Tradati N, Marazza M, Rossi N, Boracchi P, Mariani L, et al. Prevention of local relapses and new localisations of oral leukoplakias with the synthetic retinoid fenretinide (4-HPR). Preliminary results. European journal of cancer. 1992 Oct;28B(2):97-102.

60. Benner SE, Winn RJ, Lippman SM, Poland J, Hansen KS, Luna MA, et al. Regression of oral leukoplakia with alpha-tocopherol: a community clinical oncology program chemoprevention study. Journal of the National Cancer Institute. 1993 Jan 6;85(1):44-7.

61. Vedtofte P, Holmstrup P, Hjorting-Hansen E, Pindborg JJ. Surgical treatment of premalignant lesions of the oral mucosa. International journal of oral and maxillofacial surgery. $1987 \mathrm{Dec} ; 16(6)$ : 656-64.
62. Martínez González JM PGR, Baca Pérez R, Blanco Jerez L, Del Canto Pingarrón M. Cirugía láser $\mathrm{CO}_{2}$ en la eliminación de leucoplasias de la cavidad oral. Rev Eur Odontoestomatol 1993;6:321-8.

63. van der Hem PS, Nauta JM, van der Wal JE, Roodenburg JL. The results of $\mathrm{CO}_{2}$ laser surgery in patients with oral leukoplakia: a 25 year follow up. Oral oncology. 2005 Jan;41(1): 31-7.

64. Bekke JP, Baart JA. Six years' experience with cryosurgery in the oral cavity. International journal of oral surgery. 1979 Aug;8(4):251-70.

65. Gongloff RK, Samit AM, Greene GW, Jr., Inneo GF, Gage AA. Cryosurgical management of benign and dysplastic intraoral lesions. J Oral Surg. 1980 Sep;38(9):671-6.

66. Bouquot JE, Whitaker SB. Oral leukoplakiarationale for diagnosis and prognosis of its clinical subtypes or "phases". Quintessence Int. 1994 Feb;25(2):133-40.

67. Miller AB, Hoogstraten B, Staquet M, Winkler A. Reporting results of cancer treatment. Cancer. 1981 Jan 1;47(1):207-14. 